\title{
Impulse Noise Model and Its Variations
}

\author{
Haidi Ibrahim, Kuo Chue Neo, Sin Hoong Teoh, Theam Foo Ng, Derek Chan Juinn Chieh, and Nik \\ Fakhuruddin Nik Hassan
}

\begin{abstract}
Impulse noise is one of the noise types that are normally corrupting digital images. In literature, impulse noises have been described in many ways. Therefore, the main aim of this manuscript is to survey a few impulse noise models that have been used by several researchers in digital image processing field. This will lead us to a better understanding on impulse noise models, and consequently will help the researchers to design more effective impulse noise reduction filters.
\end{abstract}

Index Terms - Digital image processing, fixed-valued impulse noise, impulse noise models, median filter, random-valued impulse noise, salt-and-pepper noise.

\section{INTRODUCTION}

Currently, with the advances in electronic technologies, digital images have been used widely in our daily life. Unfortunately, digital images are sometimes corrupted by noises, which can significantly reduce the quality of the image. In general, the noise can be classified into two main groups; multiplicative noise and additive noise [1]. If $(\mathrm{x}, \mathrm{y})$ are the spatial coordinates on the image, $\mathrm{C}=\{\mathrm{C}(\mathrm{x}, \mathrm{y})\}$ is the clean and noise-free image, the damaged image $\mathrm{D}=\{\mathrm{D}(\mathrm{x}, \mathrm{y})\}$ that has been corrupted by multiplicative noise can be described as:

$$
D(x, y)=C(x, y) N(x, y)
$$

where $N=\{N(x, y)\}$ is the noise intensity value, or the noise amplitude. On the other hand, if the noise is an additive noise, generally, the damaged image can be described as:

$$
D(x, y)=C(x, y)+N(x, y)
$$

In general, additive noise is assumed to be Gaussian noise, or impulse noise [1]. However, there are a few impulse noise models available in literatures. Therefore, the purpose of this paper is to survey a few of these impulse noise models.

Manuscript received September 26, 2012; revised October 12, 2012. This work was supported in part by the Universiti Sains Malaysia's Short Term Research Grant with account number 304/PELECT/60311013.

H. Ibrahim, K. C. Neo, S. H. Teoh, T. F. Ng, and D. C. J. Chieh are with the School of Electical and Electronic Engineering, Engineering Campus, Universiti Sains Malaysia, 14300 Nibong Tebal, Penang, Malaysia (e-mail: haidi_ibrahim@ iee.org, nkc99131@@student.usm.my, tships10_eee8@student.usm.my, tfng@eng.usm.my, ch derekchan@eng.usm.my, nikf@kb.usm.my).

N. F. N. Hassan is with the Forensic Science Programme, School of Health Sciences, Health Campus, Universiti Sains Malaysia, 16150 Kubang Kerian, Kelantan, Malaysia (e-mail: nikf@kb.usm.my).

\section{IMPULSE NOISE MODELS}

This section will give descriptions on impulse noise models normally used by researchers. Degraded images corrupted by these noise models will be shown in each subsection. For this purpose, we will use the input image as shown in Fig. 1. We consider this image as the clean, uncorrupted image C. As what we can see from this figure, the structure of the objects in this image, which are the peppers, are defined clearly.

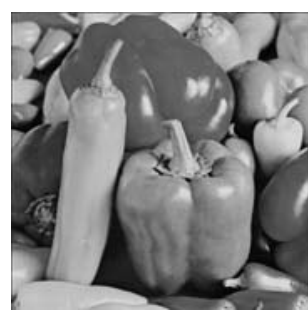

Fig. 1. An example of image that is free from impulse noise.

In general, impulse noise can be described by the following two equations. The first equation is strictly following the additive noise model, where the corrupted image $\mathbf{D}$ is defined as:

$$
D(x, y)=\left\{\begin{array}{ccc}
C(x, y) & : & \text { with probability } 1-P \\
C(x, y)+N(x, y) & : & \text { with probability } P
\end{array}\right.
$$

In this equation, $P$ (i.e. $0 \leq P \leq 1)$ presents the noise density. Higher value of $P$ means higher corruption level.

Another general impulse noise model uses a simplified version of (3). For this general model, the damaged image D is defined as:

$$
D(x, y)=\left\{\begin{array}{llc}
C(x, y) & : & \text { with probability } 1-P \\
N(x, y) & : & \text { with probability } P
\end{array}\right.
$$

This simplification is carried out because for the case of corrupted pixels, the results of $C(x, y)+N(x, y)$ in (3) can actually take any values. This is due to $N(x, y)$ is a random value. Equation (4) shows that the corrupted pixels are directly been replaced with the noise intensity values [2]-[6]. Due to this simplification, in some literatures, such as in [7],[8], impulse noise is neither considered as an additive noise, nor a multiplicative noise, but as another class of its own. Yet, unlike (3), the value of $N$ in (4) is restricted to positive values only. If the image is been quantized into $L$ intensity levels, $\mathrm{N}$ can take any values between 0 to $L-1$ [3].

\section{A. Impulse Noise Model 1}

This noise model considers that impulse noise is an additive noise, and therefore uses general equation as given by (1). This impulse noise model assumes that impulse noise 
can be characterized by a long-tail probability distribution of $N$. A long-tail probability distribution is a distribution which has relatively high probability regions far away from the mean or median values. Therefore, this model takes impulse noise as an additive long-tailed noise [9]-[11].

The probability density function $p$ of impulse noise $N$ can be modeled by some skewed distributions. Furutsu and Ishida in 1961 [9] used a combinations of Poisson distributions to present impulse noise. In 1988, Lin and Willson Jr [10] presented impulse noise by using a log-normal distribution function as given as:

$$
p(|n|)=\frac{1}{\sqrt{2 \pi|n|}} \exp \left(-\frac{\ln ^{2}(|n| / \tilde{n})}{2}\right)
$$

where $\tilde{n}=\operatorname{mean}(|n|) / \sqrt{e}$. Fig. 2 shows some degraded images, corrupted by several degree of degradation using this noise model. As shown by this figure, the impulse noise significantly lowers the quality of the image.

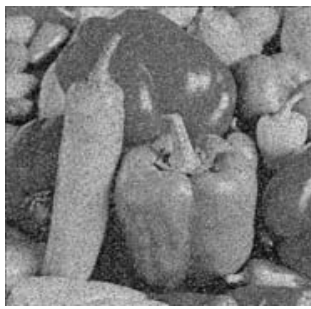

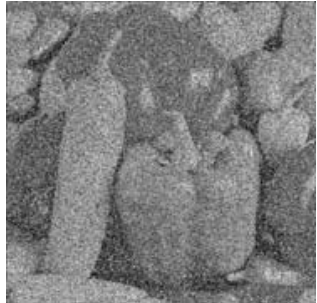

(b) (a)

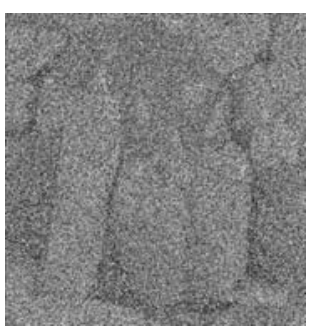

(c)

Fig. 2. Degraded versions of Fig. 1, using impulse noise model 1, with (a) $P=0.25$, (b) $P=0.50$, and (c) $P=0.75$

\section{B. Impulse Noise Model 2}

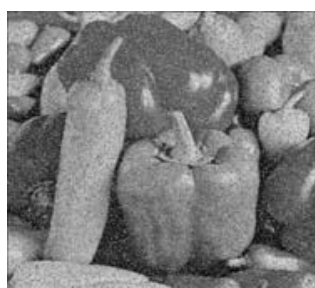

(a)

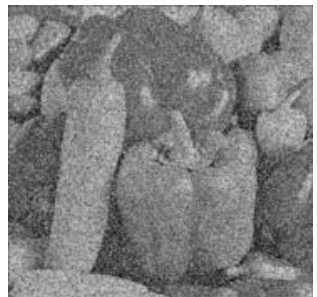

(b)

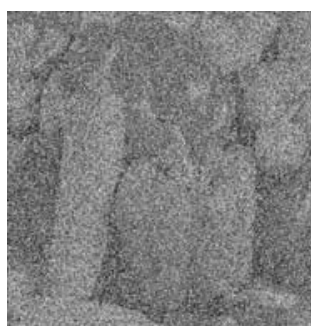

(c)
Fig. 3. Degraded versions of Fig. 1, using impulse noise model 2, with (a) $P=0.25$, (b) $P=0.50$, and (c) $P=0.75$

This noise model uses equation (4) as its general equation. For this model, the distribution $p$ of noise $N$ in (4) is defined as a uniform distribution, which can be given as:

$$
p(N)=P / L \quad 0 \leq N \leq L-1
$$

For this case, the noise amplitudes can occupy all possible intensity values, from 0 to $L-1$. This type of impulse noise is widely known as random-valued impulse noise. Random-valued impulse noise has been studied in many works, such as [12]-[16]. An example showing the image corrupted by several level of impulse noise from this model is shown in Fig. 3.

\section{Impulse Noise Model 3}

Other widely used practical impulse noise model is known as fixed-valued impulse noise. In this model, $N$ in (4) is restricted to the minimum or the maximum intensity value (i.e. 0 or $L-1$ ). It is easier to understand the idea behind this noise model by using equation (3). In (3), impulse noise $N$ can take positive or negative value. It is assumed that the magnitude of $N$ is very large, such that $C(x, y)+N(x, y)$ will produce values either much greater than $L-1$, or much lower than 0 . Due to quantization process, these values are truncated to $L-1$ or 0 [17].

As the noise with intensity 0 appears as black pixels on the image, this noise is referred as pepper noise. On the other hand, the noise with intensity $L-1$ appears as white pixels on the image. This type of noise is referred as salt noise. Therefore, fixed-valued impulse noise is also known as salt-and-pepper noise. Sometimes, this type of noise also been referred as data-drop-out or spike noise [17].

In this impulse noise model, the distribution $p$ of noise $N$ in (4) is defined as:

$$
p(N)=\left\{\begin{array}{rlc}
0.5 P & : & \text { pepper } ; N=0 \\
1-P & : & \text { noise free pixels } ; 0 \leq N \leq L-1 \\
0.5 P & : & \text { salt } ; N=L-1
\end{array}\right.
$$

Because of its simplicity and practicality, this noise model is the most popular impulse noise model used in literatures, such as works in [18]-[26]. Example of images corrupted by impulse noise using this noise model is shown in Fig. 4.

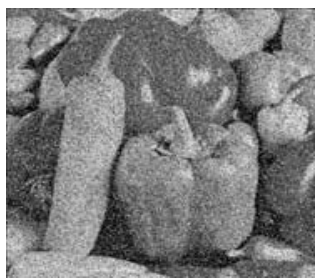

(a)

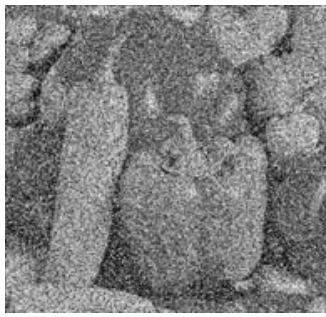

(b)

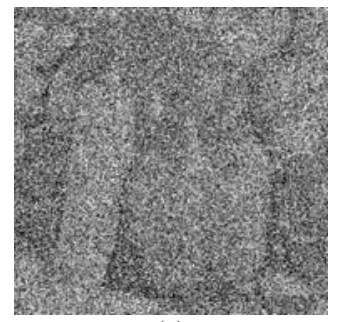

(c)
Fig. 4. Degraded versions of Fig. 1, using impulse noise model 3, with (a) $P=0.25$, (b) $P=0.50$, and (c) $P=0.75$

\section{Impulse Noise Model 4}

This noise model is achieved by modifying (7) so that the 
noise does not have equal density of salt noise and pepper noise. This can be described by the following equation:

$$
p(N)=\left\{\begin{array}{ccc}
P_{1} & : & \text { pepper } ; N=0 \\
1-P & : & \text { noise free pixels; } 0 \leq N \leq L-1 \\
P_{2} & : & \text { salt } N=L-1
\end{array}\right.
$$

where $P_{1}+P_{2}=P$, and $P_{1} \neq P_{2}$. This noise model has been used in some recent literatures, such as the works in [27]-[30]. Fig. 5 displays images corrupted by this impulse noise model. When either $P_{1}$ or $P_{2}$ is equal to zero, this type of noise is called uni-polar [17].

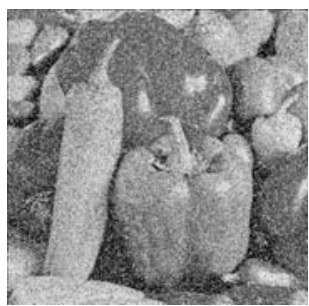

(a)

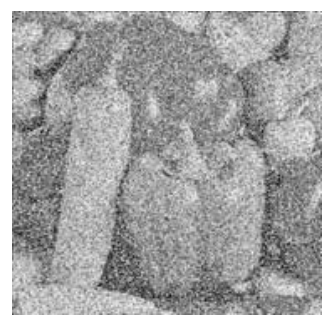

(b)

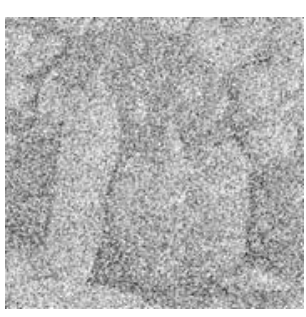

(c)
Fig. 5. Degraded versions of Fig. 1, using impulse noise model 4, with $P_{1}: P_{2}=1: 3$ and with (a) $P=0.25$, (b) $P=0.50$, and (c) $P=0.75$

\section{E. Impulse Noise Model 5}

A variation to (8) is obtained by allowing salt noise and pepper noise to be presented by two intensity ranges. Salt noise occupies high intensity range, while pepper noise occupies low intensity range. Each range is presented by $m$ intensity levels. This impulse noise model is given as:

$$
p(N)=\left\{\begin{array}{llr}
P_{1} / m & : & \text { pepper } ; 0 \leq N<m \\
1-P & : & \text { noise free pixels; } 0 \leq N \leq L-1 \\
P_{2} / m & : & \text { salt } L-1-m<N \leq L-1
\end{array}\right.
$$

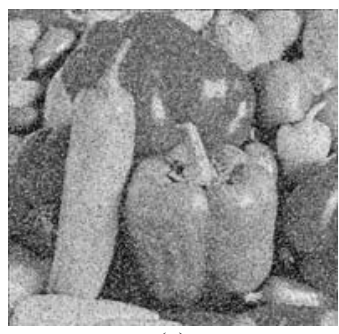

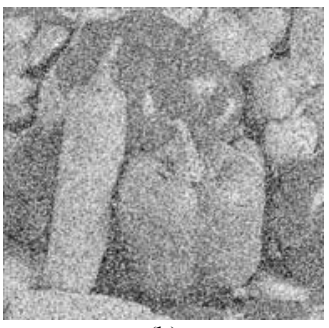

(b)

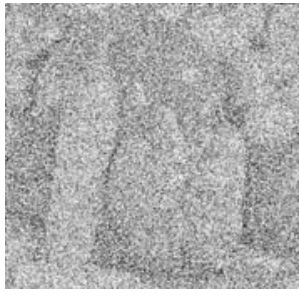

(c)
Fig. 6. Degraded versions of Fig. 1, using impulse noise model 5, with $P_{1}: P_{2}=1: 3, m=20$ and with (a) $P=0.25$, (b) $P=0.50$, and (c) $P=0.75$

Some example of works that are using this impulse noise model can be found in [27]-[30]. Example of images corrupted by impulse noise using this noise model is shown in Fig. 6.

\section{F. Impulse Noise Model 6}

Equation (9) can be simplified by assuming that the density of salt noise is equal to the density of pepper noise. This is given as:

$$
p(N)=\left\{\begin{array}{clr}
P / 2 m & : & \text { pepper } ; 0 \leq N<m \\
1-P & : & \text { noise free pixels; } 0 \leq N \leq L-1 \\
P / 2 m & : & \text { salt }[-1-m<N \leq L-1
\end{array}\right.
$$

Actually, when $m$ is equal to one, this equation is equivalent to (7), which is fixed-valued impulse noise. On the other hand, when $m$ is equal to $L / 2$, this noise model resembles (6), which is random-valued impulse noise. This noise model has been used in [27]-[30]. Fig. 7 displays images corrupted by this impulse noise model.

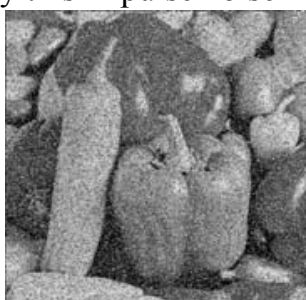

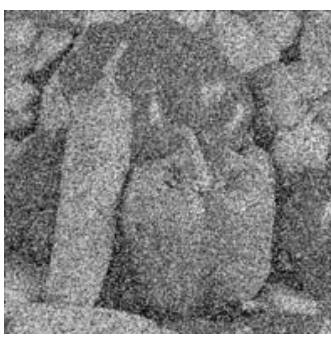

(b) (a)

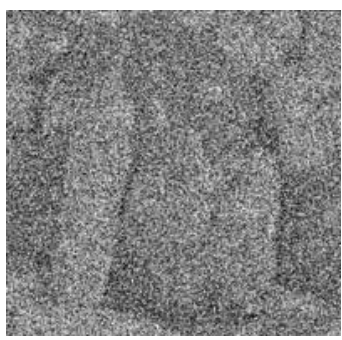

(c)
Fig. 7. Degraded versions of Fig. 1, using impulse noise model 6 , with $m=20$ and with (a) $P=0.25$, (b) $P=0.50$, and (c) $P=0.75$

\section{G. Impulse Noise Model 7}

Universal impulse noise, or also known as mixed impulse noise, is given as a combination between random-valued impulse noise defined by (6), with fixed-valued impulse noise defined by (7). In this noise model, the contamination of the image is equally contributed by these two well-known impulse noise models, which is $0.5 P$ random-valued impulse noise, and $0.5 P$ fixed-valued impulse noise. Some works related to universal impulse noise can be found in [31], [32].

\section{SUMMARY}

This paper surveys seven impulse noise models. Impulse noise model 1 is the most complicated to be generated. On the other hand, impulse noise model 3 is the simplest. From this survey, we found that all impulse noise models, excluding model 1 , must include intensity 0 and $L-1$ into their noise intensity levels.

\section{REFERENCES}

[1] M. Petrou and P. Bosdogianni, Image Processing: The Fundamentals, John Wiley and Sons, England, 1999.

[2] S. Schulte, V. D. Witte, M. Nachtegael, D. V. D. Weken, and E. E. Kerre, "A new fuzzy multi-channel filter for the reduction of impulse 
noise," in Pattern Recognition and Image Analysis, Lecture Notes in Computer Science, J. Marques, N. Perez D. L. Blanca, and P. Pina, editors, Springer Berlin/Heidelberg, 2005.

[3] S. F. Liang, S. M. Lu, J. Y. Chang, and C. T. Lee, "A novel two-stage impulse noise removal technique based on neural networks and fuzzy decision," IEEE Transactions on Fuzzy Systems, vol. 16, no. 4, pp. 863-873, 2008

[4] V. Saradhadevi and V. Sundaram, "A novel two-stage impulse noise removal technique based on neural networks and fuzzy decision," International Journal of Computer Applications, vol. 21, no. 3, pp. 31-42, May 2011.

[5] A. S. Awad and H. Man, "Similar neighbor criterion for impulse noise removal in images," AEU-International Journal of Electronics and Communications, vol. 64, no. 10, pp. 904-915, October 2010.

[6] Y. Wan, Q. Chen, and Y. Yang, "Robust impulse noise variance estimation based on image histogram," IEEE Signal Processing Letters, vol. 17 , no. 5 , May 2010 .

[7] T. Acharya and A. K. Ray, Image Processing: Principles and Applications, John Wiley \& Sons, New Jersey, 2005.

[8] S. Jayaraman, S. Essakkirajan, and T. Veerakumar, Digital Image Processing, Tata McGraw-Hill Education, New Delhi, 2009.

[9] K. Furutsu and T. Ishida, "On the theory of amplitude distribution of impulsive random noise," Journal of Applied Physics, vol. 32, no. 7, pp. 1206-1221, July 1961.

[10] H. M. Lin and A. N. W. Jr, "Median filters with adaptive length," IEEE Transactions on Circuits and Systems, vol. 35, no. 6, pp. 675-690, June 1988.

[11] E. Srinivasan and D. Ebenezer, "New nonlinear filtering strategies for eliminating short and long tailed noise in images with edge preservation properties," International Journal of Information and Communication Engineering, vol. 4, no. 3, pp. 175-181, 2008.

[12] R. H. Chan, C. Hu and M. Nikolova, "An iterative procedure for removing random-valued impulse noise," IEEE Signal Processing Letters, vol. 11, no. 12, pp. 921-924, 2004.

[13] R. Pandey, "An improved switching median filter for uniformly distributed impulse noise removal," in Proceedings of World Academy of Science, Engineering and Technology, vol. 38, pp. 349-351, 2008.

[14] P. Civicioglu, "Removal of random-valued impulsive noise from corrupted images," IEEE Transactions on Consumer Electronics, vol. 55, no. 4, pp. 2097-2104, November 2009.

[15] R. Kubota and N. Suetake, "Distribution distance-based threshold auto-tuning method for switching median filter," IEICE Electronics Express, vol. 7, no. 17, pp. 1310-1316, 2010.

[16] J. Wu and C. Tang, "PDE-based random-valued impulse noise removal based on new class of controlling functions," IEEE Transactions on Image Processing, vol. 20, no. 9, pp. 2428-2438, September 2011.

[17] R. 1. C. Gonzalez and R. E. Woods, Digital Image Processing, Pearson Prentice Hall, New Jersey, $3^{\text {rd }}$ Edition, 2008.

[18] H. Ibrahim, N. S. P. Kong and T. F. Ng, "Simple adaptive median filter for the removal of impulse noise from highly corrupted images," IEEE Transactions on Consumer Electronics, vol. 54, no. 4, pp. 1920-1927, 2008.
[19] N. S. P. Kong and H. Ibrahim, "The effect of shape and weight towards the performance of simple adaptive median filter in reducing impulse noise level from digital images, in $2^{\text {nd }}$ International Conference on Education Technology and Computer (ICETC), vol. 5, pp. 118-121, 2010 .

[20] H. Ibrahim, "Adaptive switching median filter utilizing quantized window size to remove impulse noise from digital images," Asian Transactions on Fundamentals of Electronics, Communication \& Multimedia, vol. 2, no. 1, pp. 1-6, March 2012.

[21] H. Ibrahim, T. F. Ng, and S. H. Teoh, "An efficient implementation of switching median filter with boundary discriminative noise detection for image corrupted by impulse noise," Scientific Research and Essays, vol. 6 , no. 26, pp. 5523-5533, November 2011.

[22] V. Saradhadevi and W. Sundaram, "An adaptive fuzzy switching filter for images corrupted by impulse noise," Global Journal of Computer Science and Technology, vol. 11, no. 4, pp. 29-33, March 2011.

[23] Y. Huang, M. Xie, W. Li, and L. Zhou, "Research on k-means median filter," Journal of Information \& Computational Science, vol. 8, no. 6 , pp. 961-968, 2011

[24] V. Jayaraj, D. Ebenezer, and V. R. Vijayakumar, "A noise free estimation switching median filter for detection and removal of impulse noise in images," European Journal of Scientific Research, vol. 51, no. 4, pp. 563-581, 2011.

[25] S. Essakkirajan, T. Veerakumar, A. N. Subramanyam, and C. H. Premchand, "Removal of high density salt and pepper noise through modified decision based unsymmetric trimmed median filter," IEEE Signal Processing Letters, vol. 18, no. 5, pp. 287-290, May 2011.

[26] Y. Song, Y. Han, and S. Lee, "Pixel correlation-based impulse noise reduction," in $201117^{\text {th }}$ Korea-Japan Joint Workshop on Frontiers of Computer Vision (FCV), pp. 1-4, 2011

[27] K. K. Anisha and M. Wilscy, "Impulse noise removal from medical images using fuzzy genetic algorithm," Signal \& Image Processing: an International Journal (SIPIJ), vol. 2, no. 4, pp. 173-186, 2011.

[28] C. H. Hsieh, P. C. Huang, and S. Y. Hung, "Noisy image restoration based on boundary resetting BDND and median filtering with smallest window," WSEAS Transactions on Signal Processing, vol. 5, no. 5, pp. 178-187, May 2009.

[29] P. E. Ng and K. K. Ma, "A switching median filter with boundary discriminative noise detection for extremely corrupted images," IEEE Transactions on Image Processing, vol. 15, no. 6, pp. 1506-1516, June 2006.

[30] A. K. Tripathi, U. Ghanekar, and S. Mukhopadhyay, "Switching median filter: Advanced boundary discriminative noise detection algorithm", IET Image Processing, vol. 5, no. 7, pp. 598-610, 2011.

[31] N. I. Petrovic and V. Crnojevic, "Universal impulse noise filter based on genetic programming," IEEE Transactions on Image Processing, vol. 17, no. 7, pp. 1109-1120, July 2008.

[32] A. Hussain, M. A. Jaffar, and A. M. Mirza, "A hybrid image restoration approach: Fuzzy logic and directional weighted median based uniform impulse noise removal," Knowledge and Information Systems, vol. 24 , no. 1, pp. 77-90, July 2010. 